\title{
Preservation of Blood Pressure Stability with Hypertonic Mannitol during Hemodialysis Initiation
}

\author{
Finnian R. Mc Causland ${ }^{a, b}$ Lisa M. Prior ${ }^{d}$ Eliot Heher ${ }^{b, c}$ Sushrut S. Waikar ${ }^{a, b}$ \\ ${ }^{a}$ Renal Division, Department of Medicine, Brigham and Women's Hospital, b Harvard Medical School, and \\ 'Division of Nephrology, Department of Medicine, Massachusetts General Hospital, Boston, Mass., USA; \\ dUniversity College Dublin School of Medicine and Medical Science, Dublin, Ireland
}

\section{Key Words}

Hemodialysis · Intradialytic hypotension • Mannitol •

Osmolality

\begin{abstract}
Background: Intradialytic hypotensive events are common among hemodialysis patients and are associated with a variety of patient- and procedure-related factors, including intradialytic decline in plasma osmolality. Prior studies and practice have suggested that administration of osmotically active drugs may ameliorate blood pressure decline during chronic hemodialysis. Methods: Clinical and treatment data were collected for 102 consecutive patients requiring initiation of renal replacement therapy in 2 major teaching hospitals. Routine administration of mannitol differed according to institutional protocols, allowing its examination as the primary exposure of interest. Generalized linear models were fit to estimate associations of mannitol use during dialysis initiation with intradialytic blood pressure, as assessed by: (1) intradialytic blood pressure decline; (2) nadir intradialytic blood pressure; (3) absolute systolic blood pressure $<90 \mathrm{~mm}$ $\mathrm{Hg}$ or decline $>20 \mathrm{~mm} \mathrm{Hg}$. Results: Mean age was 62 years ( \pm 16$), 70 \%$ were male and $44 \%$ were diabetic. Mean predi-
\end{abstract}

alysis and nadir systolic blood pressure were $142 \mathrm{~mm} \mathrm{Hg}$ ( \pm 29$)$ and $121 \mathrm{~mm} \mathrm{Hg}( \pm 26)$, respectively. Mannitol administration was associated with a lesser decline in intradialytic blood pressure, a higher nadir blood pressure and fewer hypotensive events requiring intervention. No effect modification was evident according to diabetes or acuity of kidney disease (chronic vs. acute). Conclusions: Mannitol administration appears to preserve hemodynamic stability during hemodialysis initiation. Randomized controlled trials are needed to confirm these findings and identify optimal management strategies to prevent intradialytic hypotension.

Copyright $\odot 2012$ S. Karger AG, Basel

\section{Introduction}

Since hemodialysis became available as a treatment modality, patients have experienced a myriad of adverse symptoms, often leading to reduced treatment efficacy and compliance. Advances in technology, dialysate composition and dialysis treatment prescriptions have only been partially successful in reducing the frequency and severity of these complaints. Intradialytic hypotensive events (IDH) in particular remain common, affecting up

\section{KARGER \\ Fax +4161306 1234 \\ E-Mail karger@karger.ch}

www.karger.com
(C) 2012 S. Karger AG, Basel

0250-8095/12/0362-0168\$38.00/0

Accessible online at:

www.karger.com/ajn
Finnian McCausland, MB, BCh

MRB-4, Brigham and Women's Hospital

Boston, MA 02446 (USA)

E-Mailfmccausland@partners.org 
to one third of all chronic hemodialysis treatments [1-3]. IDH has been associated with the development of myocardial stunning [4], cerebral atrophy [5] and increased mortality [6] and may therefore represent an important modifiable risk factor in the hemodialysis population.

Early physicians noted an association between IDH and osmolality decline during hemodialysis; preliminary evidence suggested that administration of hyperosmolar fluid (e.g., hypertonic glucose or hypertonic saline) ameliorated excessive drops in blood pressure and other dialysis-associated symptoms, presumably by preventing net fluid movement from the intravascular to the intracellular compartments [7]. However, many of these interventions resulted in unwanted side effects, including elevated blood sugar with hypertonic glucose, and increased thirst [8] and interdialytic weight gain [9] with hypertonic saline. Recent research has suggested that hyperosmolar fluid stimulates vasopressin release, which may have a direct effect on vascular tone, thereby providing an additional mechanism for intradialytic blood pressure support [10].

Mannitol is a six-carbon sugar derived from dextrose, with a molecular weight of $182 \mathrm{Da}$, undergoing predominantly renal clearance [11]. Numerous historical studies have reported an association between the use of hypertonic mannitol and reduction of intradialytic symptoms and frequency of hypotensive events in chronic hemodialysis, with seemingly fewer reported side effects compared to other interventions [12-14]. However, these were generally small physiological studies limited by sample size. Despite promising results, mannitol is not routinely used in most centers to prevent excessive intradialytic blood pressure decline. We therefore investigated the potential benefit of mannitol administration during hemodialysis initiation by comparing intradialytic hemodynamic changes between patients treated at two separate major academic teaching hospitals, one of which does and one which does not routinely administer mannitol during hemodialysis initiation.

\section{Methods}

\section{Study Design and Population}

This observational study was approved by the Partners Healthcare Institutional Review Board. Hemodialysis initiation for acute kidney injury or progressive chronic kidney disease was performed at dedicated units in Brigham and Women's Hospital (BWH; 793 beds) or Massachusetts General Hospital (MGH; 1,057 beds), both in Boston, Mass., USA. Detailed data from the first three dialysis sessions were collected from consecutive patients initiating hemodialysis between March 22nd and Decem- ber 14th, 2010. Complete data for two sessions were available in $96 \%$ of cases; complete data for 3 sessions were available for $79 \%$ of cases. There were no major differences in the proportion of missing data according to acuity of renal injury $(\mathrm{p}=0.65)$, presence of diabetes $(p=0.35)$ or heart failure $(p=0.06)$. Per institutional protocol at MGH, mannitol was administered intravenously during the dialysis session at a dose of $25 \mathrm{~g} / \mathrm{h}$; patients at $\mathrm{BWH}$ did not receive mannitol. We excluded those requiring pressors and those transitioning from continuous renal replacement therapy. The final cohort consisted of 102 individuals (278 hemodialysis sessions; $91 \%$ were performed as an inpatient).

\section{Exposures and Outcomes}

The primary outcome of interest was the decline in blood pressure during dialysis (predialysis BP - nadir intradialytic BP). Secondary outcomes included (1) the nadir intradialytic BP and (2) the development of an intradialytic hypotensive event (defined as a drop in SBP of $>20 \mathrm{~mm} \mathrm{Hg}$ or absolute SBP $<90 \mathrm{~mm} \mathrm{Hg}$ ). Blood pressure was measured using DASH 4000 monitors (GE Medical Systems; www.gemedical.com) at MGH and the internal blood pressure module of Fresenius $2008 \mathrm{~K}$ dialysis machines at $\mathrm{BWH}$ (Fresenius Medical Care North America, www.fmcna.com). Blood pressure measurements were acquired predialysis, postdialysis and every $15 \mathrm{~min}$ during the procedure. According to standard hospital protocols, all devices were calibrated on at least an annual basis.

The administration of mannitol during hemodialysis was the primary exposure of interest and was verified from nursing notes and hospital pharmacy records.

\section{Hemodialysis Prescription}

Fresenius 2008K dialysis machines and F180 membranes were used in all treatments (Fresenius Medical Care North America). Dialysis treatment parameters (time, blood and dialysate flow rate, dialysate composition and ultrafiltration rate) were prescribed at the discretion of the treating nephrologist. The dialysate sodium concentration was $140 \mathrm{mmol} / \mathrm{l}$ in $97.5 \%$ of sessions (only one treatment session had a fixed dialysate sodium concentration of $>140 \mathrm{mmol} / \mathrm{l}$ ); no patient was prescribed a sodiummodeling algorithm. Cooled dialysate was recorded in 6 sessions $\left(35-36^{\circ} \mathrm{C}\right)$. The dialysis nurse assigned to each individual subject recorded hemodynamic parameters, treatment parameters, interventions and administration of medications/fluids on standard clinical dialysis run-sheets.

\section{Study Data}

All study data were obtained via detailed paper chart and electronic medical record review. Demographic data including sex, race and age were recorded at dialysis initiation. Other variables of interest included antihypertensive medication use, comorbidities including diabetes mellitus and congestive heart failure; dialysis treatment and hemodynamic parameters; and laboratory test results (prior to each dialysis session). All laboratory tests were performed at the corresponding hospital clinical laboratory of each dialysis unit.

\section{Statistical Analysis}

Continuous variables were examined graphically and recorded as means ( \pm SDs) for normally distributed data, or medians (with inter-quartile ranges) for non-normally distributed data. 
Table 1. Baseline characteristics according to mannitol administration

\begin{tabular}{|c|c|c|c|}
\hline & $\begin{array}{l}\text { No mannitol } \\
(\mathrm{n}=54)\end{array}$ & $\begin{array}{l}\text { Mannitol } \\
(\mathrm{n}=48)\end{array}$ & $\mathrm{p}^{\mathrm{a}}$ \\
\hline Male, \% & 66.7 & 72.9 & 0.49 \\
\hline Age, years & $63.7 \pm 13.9$ & $60.4 \pm 17.3$ & 0.29 \\
\hline Black, \% & 18.5 & 10.4 & 0.25 \\
\hline DM, \% & 50.0 & 37.5 & 0.20 \\
\hline $\mathrm{CHF}, \%$ & 24.1 & 20.8 & 0.70 \\
\hline Predialysis weight, $\mathrm{kg}$ & $83.6 \pm 19.5$ & $83.6 \pm 23.3$ & $>0.90$ \\
\hline \multicolumn{4}{|l|}{ Access, $\%$} \\
\hline AVF & 30.8 & 14.9 & 0.06 \\
\hline AVG & 0.0 & 2.1 & 0.29 \\
\hline Catheter & 69.2 & 83.0 & 0.11 \\
\hline Mean predialysis SBP, $\mathrm{mm} \mathrm{Hg}$ & $141.3 \pm 30.6$ & $141.8 \pm 26.7$ & $>0.90$ \\
\hline Mean predialysis DBP, $\mathrm{mm} \mathrm{Hg}$ & $72.3 \pm 17.6$ & $70.4 \pm 16.5$ & 0.59 \\
\hline Serum sodium, $\mathrm{mmol} / \mathrm{l}$ & $135.6 \pm 5.4$ & $137.4 \pm 6.6$ & 0.15 \\
\hline Serum albumin, $\mathrm{g} / \mathrm{dl}$ & $3.2 \pm 0.7$ & $3.4 \pm 0.7$ & 0.21 \\
\hline Serum creatinine, $\mathrm{mg} / \mathrm{dl}$ & $7.1 \pm 3.5$ & $7.5 \pm 3.6$ & 0.59 \\
\hline Hemoglobin, g/dl & $9.1 \pm 1.4$ & $9.5 \pm 1.8$ & 0.26 \\
\hline Any BP medication, \% & 83.3 & 72.9 & 0.20 \\
\hline ESKD, \% & 72.2 & 56.2 & 0.09 \\
\hline UF volume, liters & $0.8(0.0,1.0)$ & $0.8(0.0,1.5)$ & 0.85 \\
\hline \multicolumn{4}{|c|}{$\begin{array}{l}\text { a } p \text { value for difference; significance testing was by } \mathrm{t} \text { test or Wilcoxon rank-sum test for continuous variables } \\
\text { and } \chi^{2} \text { test for dichotomous variables. } \\
\text { Parameters are presented as mean } \pm \text { standard deviation or median (interquartile range). } \\
\qquad \mathrm{DM}=\mathrm{Diabetes} \text { mellitus; } \mathrm{CHF}=\mathrm{congestive} \text { heart failure; } \mathrm{AVF}=\text { arteriovenous fistula; } \mathrm{AVG}=\text { arteriovenous } \\
\text { graft; } \mathrm{SBP}=\text { systolic blood pressure; } \mathrm{DBP}=\text { diastolic blood pressure; } \mathrm{ESKD}=\text { end-stage kidney disease (vs. ref- } \\
\text { erence of acute kidney injury requiring hemodialysis); UF = ultrafiltration at first session. }\end{array}$} \\
\hline
\end{tabular}

Comparisons were made using t tests or Wilcoxon rank sum tests, as appropriate. Categorical variables were examined by frequency distribution, recorded as proportions and comparisons made using the $\chi^{2}$ test.

The association of mannitol use (vs. standard treatment) with hemodynamic outcomes of interest was examined by fitting generalized linear regression models, with and without adjustment for case mix. Each individual could potentially contribute data from three separate dialysis sessions; the use of a repeated measures design was therefore required, in which clustered variance estimates were used to account for non-independence of covariates within subject, thereby allowing more accurate estimation of the standard error related to our exposure of interest. Individual covariates were selected on the basis of clinical and biological plausibility, without use of probabilistic selection criteria. Logarithmic transformation was performed for outcomes of interest that were found to be non-normally distributed.

Effect modification of mannitol and outcome according to prespecified variables of interest (diabetes, heart failure and acuity of kidney disease) was assessed by the inclusion of cross-product terms. Nominal two-sided p values of $<0.05$ were considered statistically significant. Analyses were performed using SAS v9.2 (SAS Institute, Carey, N.C., USA) and STATA 10.0MP (College Station, Tex., USA).

\section{Results}

\section{Baseline Characteristics}

The primary cohort consisted of 102 patients; 48 (47\%; all at $\mathrm{MGH}$ ) received mannitol during their initiation treatments; 54 (53\%; all at $\mathrm{BWH})$ did not receive mannitol. Mean age was $62.1( \pm 15.6)$ years, $44.1 \%$ were diabetic, $22.6 \%$ had a history of congestive heart failure. There were no differences between those who did and did not receive mannitol according to age, sex, race, diabetes, heart failure, serum sodium, serum creatinine, antihypertensive use or predialysis blood pressure at baseline. The mannitol group contained a greater proportion of patients who initiated hemodialysis for acute indications and had dialysis catheters, though these differences were not statistically significant (table 1). Modest clinical differences in session length, blood and dialysate flow were evident both between groups and according to dialysis session chronology (table 2). 


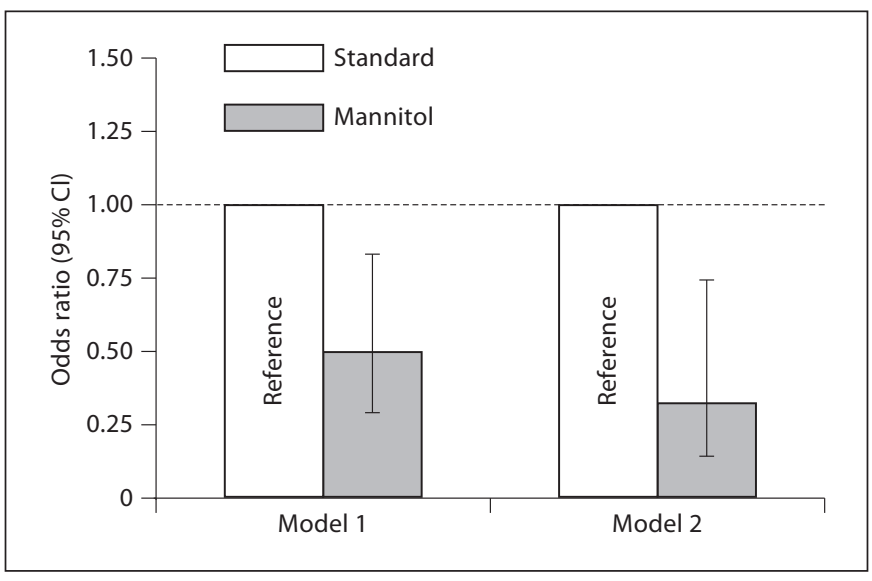

Fig. 1. Association of mannitol (gray bar) versus no mannitol administration (reference; white bar) with a drop of $>20 \mathrm{~mm} \mathrm{Hg}$ from predialysis systolic blood pressure (SBP) or minimum intradialytic SBP $<90 \mathrm{~mm} \mathrm{Hg}$. Estimates from model 1 were adjusted for age, sex, diabetes, access (catheter vs. noncatheter), predialysis SBP and congestive heart failure. Model 2 was additionally adjusted for predialysis serum sodium, predialysis weight, acuity of kidney injury (chronic vs. acute) and categories of ultrafiltration $(0,0-1,1-2$ and $>21)$, session length $(\leq 120,121-180,>180 \mathrm{~min})$, blood flow $(<300,300-399,400 \mathrm{ml} / \mathrm{min})$ and dialysate flow $(\leq 500,501-600,601-800 \mathrm{ml} / \mathrm{min})$.

\section{Association of Mannitol Use with Nadir Blood}

Pressure during Dialysis and Drop in Blood Pressure

There was no significant difference in the mean unadjusted predialysis SBP over the initiation sessions for those patients who received mannitol versus those who did not $(\mathrm{p}=0.9)$, nor in the mean unadjusted intradialytic nadir SBP $(\mathrm{p}=0.11)$. However, in case-mix-adjusted models (adjusted for age, sex, diabetes, congestive heart failure, catheter use and predialysis SBP), patients treated with mannitol had $5.4 \mathrm{~mm} \mathrm{Hg}$ higher nadir intradialytic SBP compared to those who were not ( $\mathrm{p}=0.03$; table 3$)$. In exploratory models, additional adjustment for predialysis sodium, predialysis weight, acuity of kidney disease, UF volume, session length, blood flow and dialysate flow resulted in accentuation of the effect estimate (8.2 mm Hg higher nadir SBP with mannitol; $\mathrm{p}=0.01$ ).

Systolic BP decline (predialysis SBP - SBP nadir) was considered as a log-transformed variable due to non-normal distribution. Individuals treated with mannitol had $30.0 \%$ and $25.1 \%$ less decline in log SBP in unadjusted $(\mathrm{p}=0.02)$ and adjusted $(\mathrm{p}=0.03)$ analyses, respectively. In exploratory models, additional adjustment for predialysis sodium, predialysis weight, acuity of kidney disease, UF volume, session length, blood flow and dialysate
Table 2. Dialysis treatment parameters according to mannitol administration

\begin{tabular}{lllc}
\hline & $\begin{array}{l}\text { No mannitol } \\
(\mathrm{n}=54)\end{array}$ & $\begin{array}{l}\text { Mannitol } \\
(\mathrm{n}=48)\end{array}$ & $\mathrm{p}^{\mathrm{a}}$ \\
\hline $\begin{array}{l}\text { Session length, min } \\
\text { 1st session }\end{array}$ & $120(120,120)$ & $150(120,180)$ & $<0.001$ \\
2nd session & $180(180,180)$ & $180(180,180)$ & 0.41 \\
3rd session & $240(210,240)$ & $210(180,240)$ & 0.01 \\
Blood flow, ml/min & & & \\
1st session & $200(200,200)$ & $250(200,250)$ & $<0.001$ \\
2nd session & $300(300,300)$ & $300(250,300)$ & 0.01 \\
3rd session & $400(400,400)$ & $350(300,400)$ & $<0.001$ \\
Dialysate flow, ml/min & & & \\
1st session & $400(400,400)$ & $500(500,500)$ & $<0.001$ \\
2nd session & $600(600,600)$ & $500(500,600)$ & $<0.001$ \\
3rd session & $800(800,800)$ & $800(600,800)$ & $<0.001$ \\
\hline
\end{tabular}

${ }^{\mathrm{a}} \mathrm{p}$ value for difference; significance testing was by Wilcoxon rank-sum test.

Parameters are presented as median (interquartile range).

flow demonstrated that mannitol use was associated with $27 \%$ less decline in log SBP ( $p=0.047)$. There was no evidence for effect modification according to the presence of diabetes, heart failure or acuity of kidney disease ( $\mathrm{p}$ interaction $>0.10$ ).

\section{Association of Mannitol Use with Intradialytic \\ Hypotensive Events}

Overall, $51 \%$ of sessions were complicated by a hypotensive event, defined in this study as a drop of $20 \mathrm{~mm} \mathrm{Hg}$ in SBP or absolute SBP $<90 \mathrm{~mm} \mathrm{Hg}$. In adjusted models (age, sex, diabetes, congestive heart failure, catheter use and predialysis SBP), patients treated with mannitol had $50 \%$ lower odds of experiencing hypotensive events (OR 0.50 ; 95\% CI 0.29-0.83). In exploratory models, upon additional adjustment for predialysis sodium, predialysis weight, acuity of kidney disease, UF volume, session length, blood flow and dialysate flow, the effect estimate was accentuated and remained significant (OR 0.32; 95\% CI 0.14-0.74; fig. 1).

In further sensitivity analyses, after modifying the definition of a hypotensive event (drop in SBP of $>40 \mathrm{~mm}$ $\mathrm{Hg}$ or absolute SBP nadir $<90 \mathrm{~mm} \mathrm{Hg}$; which occurred in $28 \%$ of sessions), the use of mannitol remained associated with lower case-mix-adjusted odds of developing a hypotensive event (OR 0.35; 95\% CI 0.18-0.66). 
Table 3. Unadjusted and case-mix-adjusted associations between mannitol administration and hemodynamic parameters

\begin{tabular}{lllll}
\hline & \multicolumn{2}{l}{ Difference for mannitol (vs. no mannitol) } & \\
\cline { 2 - 5 } & unadjusted & $\mathrm{p}$ & adjusted $^{\mathrm{b}}$ & $\mathrm{p}$ \\
\hline Predialysis SBP, $\mathrm{mm} \mathrm{Hg}^{\mathrm{c}}$ & $0.6(-9.0,10.2)$ & 0.9 & $1.9(-7.3,11.2)$ & 0.68 \\
Nadir SBP, $\mathrm{mm} \mathrm{Hg}^{\mathrm{c}}$ & $6.9(-1.6,15.4)$ & 0.11 & $5.4(0.6,10.2)$ & 0.03 \\
Log drop in SBP, $\mathrm{mm} \mathrm{Hg}^{\mathrm{c}}$ & $-0.36(-0.65,-0.06)$ & 0.02 & $-0.29(-0.54,-0.03)$ & 0.03 \\
\hline
\end{tabular}

${ }^{a}$ Generalized linear models were fit using clustered variances to account for non-independence of individuals. Effect estimates presented are beta coefficients, $95 \%$ CIs and $p$ values testing for the effect of mannitol administration versus standard therapy.

${ }^{b}$ Adjusted effect estimates were estimated by adding age, sex, diabetes, access (catheter vs. noncatheter), predialysis SBP and congestive heart failure to the model. Predialysis SBP was omitted from the covariate list in the model considering it as the dependent variable.
${ }^{c}$ Exponentiation of the beta coefficient provides an estimate of the ratio of the geometric mean in SBP decline in patients treated with mannitol vs. those who did not receive mannitol. Unadjusted analysis reveals a ratio of 0.70 (30\% less decline in SBP; 13.7 vs. $19.6 \mathrm{~mm} \mathrm{Hg}$ ); adjusted analysis reveals a ratio of 0.75 (25\% less decline in SBP; 16.3 vs. $21.8 \mathrm{~mm} \mathrm{Hg}$ when considering a male, diabetic, aged 62 years, using a catheter, with heart failure and predialysis SBP of $142 \mathrm{~mm} \mathrm{Hg}$ ).

$\mathrm{SBP}=$ Systolic blood pressure.

\section{Discussion}

In this study, we have leveraged institutional differences between two large academic teaching hospitals during hemodialysis initiation to explore the effects of hypertonic mannitol on intradialytic hemodynamic parameters. We found that patients who receive hypertonic mannitol during hemodialysis initiation have: (1) higher nadir SBP during dialysis, (2) less decline in SBP during hemodialysis, and (3) lower odds of experiencing intradialytic hypotensive events.

The role of osmolality in the genesis of dialysis disequilibrium and hemodynamic sequelae has been hypothesized for many decades, with supportive evidence initially coming indirectly from clinical observations. Early dialysis treatments utilized a low dialysate sodium concentration $(\sim 127 \mathrm{mmol} / \mathrm{l})$ in order to facilitate sodium removal [15]. However, with the implementation of pressure-driven ultrafiltration (which allowed volume removal in a controlled fashion over shorter periods of time) many patients developed adverse effects that were ameliorated by raising the dialysate sodium concentration from $\sim 127$ to $\sim 140 \mathrm{mmol} / \mathrm{l}$ [15]. This concept was further developed by the use of fixed higher dialysate sodium concentrations [16] and sodium modeling algorithms $[17,18]$, which were purported to preserve intravascular volume and promote intradialytic hemodynamic stability. Older literature and clinical experience confirmed the beneficial effects of hypertonic saline, hypertonic glucose and hypertonic mannitol in the treatment of dialysis-related cramps [7].
There are sound physiological hypotheses as to why osmolality could influence hemodynamic stability during hemodialysis. A study in which hemodynamic stability was challenged (by removal of $2-3 \%$ body weight over $2 \mathrm{~h}$ during various investigational dialytic protocols), confirmed a marked decline in measured plasma osmolality during the course of hemodialysis, and noted a close correlation with the decline in mean arterial pressure (MAP). In addition to standard hemodialysis, a decline in osmolality was also observed under the experimental conditions of isokalemic dialysis and with the use of isotonic mannitol. However, no significant osmolality changes (nor changes in MAP) were noted with the protocols utilizing isolated ultrafiltration or hypertonic mannitol, supporting an association between preservation of osmolality and hemodynamic stability [14]. Left unanswered by this study is the mechanism by which higher osmolality was associated with improved hemodynamic stability. To address this, Shimuzi et al. [10] studied the hemodynamic and biochemical effects of infusions of $10 \%$ saline, $50 \%$ glucose, $0.9 \%$ saline or arginine vasopressin (AVP) during intradialytic hypotensive episodes. The investigators found that hypertonic saline infusion increased plasma osmolality (292-302 mosm/ kg; p < 0.001), plasma AVP (3.9-7.8 pg/ml; p = 0.03) and MAP (66.6-71.8 mm Hg; $\mathrm{p}=0.01)$. Although they did detect an increase in plasma volume with hypertonic saline, they postulated the magnitude (2.3\%) of the rise was not sufficient to explain the corresponding increase in MAP; instead they hypothesized a role for a vasopressive effect of AVP in blood pressure maintenance. The au- 
thors replicated their findings in a subsequent study [19], while other investigators have reported that subjects with IDH were unable to appropriately increase AVP levels in response to hemodynamic stressors [20]. A further study demonstrated that infusion of AVP alone improved cardiovascular stability during dialysis, thus lending additional evidence regarding its role in the maintenance of vascular tone [21].

We found that mannitol administration led to improved hemodynamic stability during dialysis according to a number of different metrics, including change in systolic blood pressure, nadir blood pressure and development of hypotensive events (SBP decline $>20 \mathrm{~mm} \mathrm{Hg}$ or absolute $\mathrm{SBP}<90 \mathrm{~mm} \mathrm{Hg}$ ). Our findings extend previous reports as we specifically examined the period of dialysis initiation, when the decline in osmolality caused by dialysis is perhaps greatest.

The prevention of hypotensive events deserves to be a major quality improvement target in hemodialysis, given the association with the development of cerebral atrophy [5], myocardial dysfunction [22], and increased longterm mortality [6]. Myocardial blood flow studies have detected reduced myocardial perfusion associated with hemodialysis itself [23]. It is possible that the phenomenon of myocardial stunning, exacerbated by intradialytic hypotension, may contribute to the unacceptably high 90 -day mortality rate [24] noted with hemodialysis initiations; further studies are required to investigate this possibility. There were no apparent major adverse effects of mannitol administration, though this was not systematically studied. Potential concerns may include the precipitation of pulmonary edema by mannitol, though this might be offset by better toleration of ultrafiltration. Given the potential concern for mannitol-induced acute kidney injury [25], future trials should consider measurement of residual blood mannitol levels and perform detailed examination of renal recovery between treatment arms.

A significant strength of this study was the ability to control for individual subject-level characteristics across both clinical sites. In this regard we were able to circumvent the potential pitfall of ecological fallacy, where individual members of a group are erroneously assumed to have the average characteristics of that group. We purposefully took advantage of differences in institutional protocols that allowed us to examine mannitol administration as our primary exposure of interest; in some ways this was comparable to a quasi-randomized trial design. However, without the primacy of actual randomization, we were particularly cognizant to adjust for potential differences in subject characteristics between the two centers. The lack of major differences in baseline characteristics, and statistical significance of results after adjustment for potential confounders, argues against differences in disease severity or co-morbidities as explanations for our findings.

There are several limitations of this study that merit further discussion. Limitations inherent to observational studies remain, including the potential for residual confounding based on incomplete adjustment of variables considered or from those not considered at all; of note, it is less likely that our results suffer from confounding by indication, as the initiation protocols implemented at each institution largely remove the influence of patient and physician-related factors in the decision to use mannitol. It is possible that other unmeasured differences in physician and/or nursing practice patterns, or unmeasured patient characteristics between the two institutions may account for our findings. For example, MGH has the ability to perform continuous intradialytic blood volume monitoring, whereas BWH does not. Blood volume monitoring was not found to be associated with a reduction in intradialytic hypotension in a previous randomized controlled trial [26]. Data limitations precluded us from determining the proportion of patients in whom blood volume monitoring was actually utilized and whether medical interventions occurred secondary to changes in blood volume monitoring itself. However, assuming the clinical response to blood volume monitoring would lead to alterations in achieved ultrafiltration, we did not detect attenuation of effect estimates after additional adjustment for ultrafiltration, arguing against a role for blood volume monitoring as a major confounder of our findings. A notable feature of this study was the inclusion of subjects with both acute and chronic indications for renal replacement therapy. We tested for (and excluded) effect modification based on the acuity of kidney disease. However, we recognize that these may be distinct pathophysiological processes in terms of volume homeostasis and hemodynamic stability. Overall, our sample size was modest and due to data collection limitations, we were unable to delineate the timing of hypotensive events and other associated patient symptoms at a more granular level. Generalizability may be limited because all patients were treated in two large academic teaching hospitals, in the setting of dialysis initiation.

In conclusion, our comparison of hemodynamic patterns between patients at two institutions that differ in the use of hypertonic mannitol for hemodialysis initiation suggests that hypertonic mannitol may help to pre- 
vent excessive intradialytic blood pressure decline. Additional interventional studies are required to confirm our findings in dialysis initiation, systematically assess for potential adverse effects and to investigate potential therapeutic extension to the chronic hemodialysis setting.

\section{Acknowledgements}

The authors wish to gratefully acknowledge the assistance of Dr. Nina Tolkoff-Rubin MD, Dr. Steven Brunelli MD MSCE, Ms. Nyla Shellito RN, Ms. Susan Taylor RN, Ms. Katherine Serge RN and Ms. Gail Appling for facilitating the completion of this study.

Portions of this work were accepted in abstract form for the National Kidney Foundation Spring Clinical Meeting, 2012.
This work was conducted with support from the Scholars in Clinical Science Program of Harvard Catalyst, The Harvard Clinical and Translational Science Center (Award No. UL1 RR025758 and financial contributions from Harvard University and its affiliated academic health care centers). The content is solely the responsibility of the authors and does not necessarily represent the official views of Harvard Catalyst, Harvard University and its affiliated academic health care centers, the National Center for Research Resources, or the National Institutes of Health.

\section{Disclosure Statement}

Dr. McCausland is supported by a research fellowship from the National Kidney Foundation (2011-13).

Dr. Waikar is supported by DK075941 and UO1DK085660.

\section{References}

1 Bos WJ, Bruin S, van Olden RW, Keur I, Wesseling $\mathrm{KH}$, Westerhof N, Krediet RT, Arisz LA: Cardiac and hemodynamic effects of hemodialysis and ultrafiltration. Am J Kidney Dis 2000;35:819-826.

2 Boon D, van Montfrans GA, Koopman MG, Krediet RT, Bos WJ: Blood pressure response to uncomplicated hemodialysis: the importance of changes in stroke volume. Nephron Clin Pract 2004;96:c82-c87.

-3 Palmer BF, Henrich WL: Recent advances in the prevention and management of intradialytic hypotension. J Am Soc Nephrol 2008; 19:8-11.

-4 Owen PJ, Priestman WS, Sigrist MK, Lambie SH, John SG, Chesterton LJ, McIntyre CW: Myocardial contractile function and intradialytic hypotension. Hemodial Int 2009;13: 293-300.

-5 Mizumasa T, Hirakata H, Yoshimitsu T, Hirakata E, Kubo M, Kashiwagi M, Tanaka H, Kanai H, Fujimi S, Iida M: Dialysis-related hypotension as a cause of progressive frontal lobe atrophy in chronic hemodialysis patients: a 3-year prospective study. Nephron Clin Pract 2004;97:c23-c30.

6 Shoji T, Tsubakihara Y, Fujii M, Imai E: Hemodialysis-associated hypotension as an independent risk factor for two-year mortality in hemodialysis patients. Kidney Int 2004 66:1212-1220.

-7 Canzanello VJ, Hylander-Rossner B, Sands RE, Morgan TM, Jordan J, Burkart JM: Comparison of $50 \%$ dextrose water, $25 \%$ mannitol, and $23.5 \%$ saline for the treatment of hemodialysis-associated muscle cramps. ASAIO Trans 1991;37:649-652.

-8 Sang GL, Kovithavongs C, Ulan R, Kjellstrand CM: Sodium ramping in hemodialysis: a study of beneficial and adverse effects. Am J Kidney Dis 1997;29:669-677.
-9 Song JH, Park GH, Lee SY, Lee SW, Kim MJ: Effect of sodium balance and the combination of ultrafiltration profile during sodium profiling hemodialysis on the maintenance of the quality of dialysis and sodium and fluid balances. J Am Soc Nephrol 2005;16:237246.

10 Shimizu K, Kurosawa T, Sanjo T: Effect of hyperosmolality on vasopressin secretion in intradialytic hypotension: a mechanistic study. Am J Kidney Dis 2008;52:294-304.

11 Nissenson AR, Weston RE, Kleeman CR: Mannitol. West J Med 1979;131:277-284.

12 Hagstam KE, Lindergard B, Tibbling G: Mannitol infusion in regular haemodialysis treatment for chronic renal insufficiency. Scand J Urol Nephrol 1969;3:257-263.

13 Hothi DK, Harvey E, Goia CM, Geary D: The value of sequential dialysis, mannitol and midodrine in managing children prone to dialysis failure. Pediatr Nephrol 2009;24: 1587-1591.

14 Henrich WL, Woodard TD, Blachley JD, Gomez-Sanchez C, Pettinger W, Cronin RE: Role of osmolality in blood pressure stability after dialysis and ultrafiltration. Kidney Int 1980;18:480-488.

15 Flanigan MJ: Role of sodium in hemodialysis. Kidney Int Suppl 2000;76:S72-S78.

16 Cybulsky AV, Matni A, Hollomby DJ: Effects of high sodium dialysate during maintenance hemodialysis. Nephron 1985;41:5761.

17 Sadowski RH, Allred EN, Jabs K: Sodium modeling ameliorates intradialytic and interdialytic symptoms in young hemodialysis patients. J Am Soc Nephrol 1993;4:11921198.

18 Kimura G, Van Stone JC, Bauer JH: Model prediction of plasma volume change induced by hemodialysis. J Lab Clin Med 1984;104: 932-938.
19 Shimizu K, Kurosawa T, Ishikawa R, Sanjo T: Vasopressin secretion by hypertonic saline infusion during hemodialysis: effect of cardiopulmonary recirculation. Nephrol Dial Transplant 2011;2011:7.

20 Rho M, Perazella MA, Parikh CR, Peixoto AJ, Brewster UC: Serum vasopressin response in patients with intradialytic hypotension: a pilot study. Clin J Am Soc Nephrol 2008;3:729-735

21 van der Zee S, Thompson A, Zimmerman R, Lin J, Huan Y, Braskett M, Sciacca RR, Landry DW, Oliver JA: Vasopressin administration facilitates fluid removal during hemodialysis. Kidney Int 2007;71:318-324.

-22 Selby NM, Lambie SH, Camici PG, Baker CS, McIntyre CW: Occurrence of regional left ventricular dysfunction in patients undergoing standard and biofeedback dialysis. Am J Kidney Dis 2006;47:830-841.

23 McIntyre CW, Burton JO, Selby NM, Leccisotti L, Korsheed S, Baker CS, Camici PG: Hemodialysis-induced cardiac dysfunction is associated with an acute reduction in global and segmental myocardial blood flow. Clin J Am Soc Nephrol 2008;3:19-26.

24 Soucie JM, McClellan WM: Early death in dialysis patients: risk factors and impact on incidence and mortality rates. J Am Soc Nephrol 1996;7:2169-2175.

25 Dickenmann M, Oettl T, Mihatsch MJ: Osmotic nephrosis: acute kidney injury with accumulation of proximal tubular lysosomes due to administration of exogenous solutes. Am J Kidney Dis 2008;51:491-503.

-26 Reddan DN, Szczech LA, Hasselblad V, Lowrie EG, Lindsay RM, Himmelfarb J, Toto RD, Stivelman J, Winchester JF, Zillman LA, Califf RM, Owen WF Jr: Intradialytic blood volume monitoring in ambulatory hemodialysis patients: a randomized trial. J Am Soc Nephrol 2005; 16:2162-2169. 